\title{
Avaliação da atividade antimicrobiana, antioxidante e citoprotetora da quercetina contra a ação tóxica do cloreto de bário
}

\author{
Evaluation of the antimicrobial, antioxidant and cytoprotective activity of kercetin against the toxic \\ action of barium chloride
}

Evaluación de la actividad antimicrobiana, antioxidante y citoprotectiva de la kercetina frente a la acción tóxica del cloruro de bario

Recebido: 30/04/2021 | Revisado: 06/05/2021 | Aceito: 09/05/2021 | Publicado: 22/05/2021

Francisco Eduardo Tavares Mendes
ORCID: https://orcid.org/0000-0002-1021-338X
Centro Universitário Dr. Leão Sampaio, Brasil
E-mail: tavareseduardo39@ gmail.com
Gustavo Marinho Miranda
ORCID: https://orcid.org/0000-0002-6013-054X
Fundação Oswaldo Cruz, Brasil
E-mail: gustavobiomed116@ @mail.com
Helena Kelly Vieira Sobreira Camilo
ORCID: https://orcid.org/0000-0001-5057-5548
Centro Universitário Dr. Leão Sampaio, Brasil
E-mail: helenakel1998@ gmail.com
Rivânia da Silva Lira
ORCID: https://orcid.org/0000-0001-9818-0035
E-mail: rivania.lira@ifma.edu.br
Vanessa de Carvalho Nilo Bitu
Instituto Federal de Educação, Ciência e Tecnogia do Manhão, Brasil
ORCID: https://orcid.org/0000-0003-0688-1403
E-mail: vanessanilobitu@ @mail.com
Celestina Elba Sobral de Souza
ORCID: https://orcid.org/0000- 0002-9595-5728
Centro Universitário Vale do Salgado; Brasil
Centro Universitário Dr. Leão Sampaio, Brasil
E-mail: sobralelba5@gmail.com

\begin{abstract}
Resumo
Este trabalho teve como objetivo avaliar a atividade antimicrobiana, antioxidante e citoprotetora da quercetina frente a toxicidade do cloreto de bário. A solução teste foi preparada a partir da diluição utilizando DMSO, posteriormente foram realizadas diluições a fim de obter concentrações distintas. A Concentração Inibitória Mínima (CIM) foi obtida através de microdiluições em caldo, para leitura dos ensaios com a bactéria uma solução de resazurina sódica foi adicionada em cada cavidade $(20 \mu \mathrm{L})$ e as placas ficaram em repouso por $1 \mathrm{~h}$ em temperatura ambiente. A avaliação do efeito citoprotetor da quercetina ao cloreto de bário, foram preparados eppendorfs contendo concentração subinibitória da amostra e suspensões de $10^{5} \mathrm{UFC} / \mathrm{ml}$ de Escherichia coli 06 (EC 06) e Candida albicans (CA 40006) em meio TRIS M9. A solução foi distribuída na placa de microdiluição. Em seguida foi adicionado $100 \mu \mathrm{L}$ do cloreto de bário na primeira cavidade seguindo com sucessivas microdiluições até a penúltima cavidade. A ação antioxidante da substância foi avaliada usando o método DPPH (1,1-difenil-2-picril-hidrazilo), os testes foram realizados em triplicata, observando que a quercetina apresenta eficácia na sua ação antioxidante. A quercetina não apresentou ação no CIM, com resultado de $\geq 1024 \mu \mathrm{g} / \mathrm{ml}$. Observou que a quercetina apresentou efeito citoprotetor frente a Escherichia coli, quando associado ao cloreto de bário, a quercetina apresentou resultados positivos relacionado a ação da sua atividade citoprotetora. Esses resultados abrem possibilidades para evolução de estudos complementares e a utilização em ambientes contaminados.
\end{abstract}

Palavras-chave: Antibacteriana; Antifúngica; Antioxidante; Citoprotetora; Quercetina.

\section{Abstract}

This study aimed to evaluate the antimicrobial, antioxidant and cytoprotective activity of quercetin against the toxicity of barium chloride. The test solution was prepared from the dilution using DMSO, later dilutions were made in order to obtain different concentrations. The Minimum Inhibitory Concentration (MIC) was obtained through microdilutions in broth, for reading the tests with the bacteria a solution of sodium resazurin was added in each well 
$(20 \mu \mathrm{L})$ and the plates were left to stand for $1 \mathrm{~h}$ at room temperature. To evaluate the cytoprotective effect of quercetin to barium chloride, eppendorfs were prepared containing subinhibitory concentration of the sample and suspensions of $105 \mathrm{CFU} / \mathrm{ml}$ of Escherichia coli 06 (EC 06) and Candida albicans (CA 40006) in TRIS M9 medium. The solution was distributed on the microdilution plate. Then $100 \mu \mathrm{L}$ of barium chloride was added to the first well, followed by successive microdilutions until the penultimate well. The antioxidant action of the substance was evaluated using the DPPH method (1,1-diphenyl-2-picryl-hydrazyl), the tests were carried out in triplicate, noting that quercetin is effective in its antioxidant action. Quercetin had no action on MIC, with a result of $\geq 1024 \mu \mathrm{g} / \mathrm{ml}$. He observed that quercetin had a cytoprotective effect against Escherichia coli, when associated with barium chloride, quercetin showed positive results related to the action of its cytoprotective activity. These results open possibilities for the evolution of complementary studies and the use in contaminated environments.

Keywords: Antibacterial; Antifungal; Antioxidant; Citoprotector; Quercetin.

\section{Resumen}

Este estudio tuvo como objetivo evaluar la actividad antimicrobiana, antioxidante y citoprotectora de la quercetina frente a la toxicidad del cloruro de bario. La solución de prueba se preparó a partir de la dilución utilizando DMSO, posteriormente se realizaron diluciones para obtener diferentes concentraciones. La Concentración Mínima Inhibitoria (CIM) se obtuvo mediante microdiluciones en caldo, para la lectura de las pruebas con las bacterias se añadió una solución de resazurina sódica en cada pocillo $(20 \mu \mathrm{L})$ y las placas se dejaron reposar 1 ha temperatura ambiente. Para evaluar el efecto citoprotector de la quercetina al cloruro de bario, se prepararon eppendorfs que contenían concentración subinhibitoria de la muestra y suspensiones de 105 UFC / ml de Escherichia coli 06 (EC 06) y Candida albicans (CA 40006) en medio TRIS M9. La solución se distribuyó sobre la placa de microdilución. Luego se agregaron $100 \mu \mathrm{L}$ de cloruro de bario al primer pocillo, seguido de microdiluciones sucesivas hasta el penúltimo pozo. La acción antioxidante de la sustancia se evaluó mediante el método DPPH (1,1-difenil-2-picryl-hidrazil), las pruebas se realizaron por triplicado, destacando que la quercetina es eficaz en su acción antioxidante. La quercetina no tuvo acción sobre la CMI, con un resultado de $\geq 1024 \mu \mathrm{g} / \mathrm{ml}$. Observó que la quercetina tenía un efecto citoprotector contra Escherichia coli, cuando se asoció con el cloruro de bario, la quercetina mostró resultados positivos relacionados con la acción de su actividad citoprotectora.

Palabras clave: Antibacteriano; Antifúngico; Antioxidante; Citoprotector; Quercetina.

\section{Introdução}

Os metais pesados quando expostos no meio ambiente têm a capacidade de gerar contaminação no solo e demais ecossistemas, isso pode acontecer de maneira natural e/ou antrópica chegando a ser um grande problema para o meio ambiente. Uma das características desses metais pesados é a sua capacidade de se acumular no ambiente e isso acontece pelo fato desses metais não serem biodegradáveis, e assim, responsáveis por diversas alterações como: inibir o crescimento de vegetações, inibir a atividade microbiana alterando também a biodiversidade da mesma (Lima, 2012; Martins, 2009).

Em meio a todos os metais pesados podemos citar o bário; metal que comumente não se apresenta livre no ambiente, naturalmente encontra-se como um elemento traço (ETs) constituindo rochas e sedimentares, a água pode ser um dos principais meios de transporte responsável por carrear o bário disseminando ainda mais a contaminação (Lima, Sobrinho Amaral, Magalhães, Guedes \& Zont, 2012).

A toxicidade e os efeitos nocivos do bário no organismo humano e no meio ambiente irão depender de uma série de fatores sendo avaliado principalmente a sua solubilidade, na água ou no estômago, como, por exemplo compostos como o acetato de bário onde o mesmo é solúvel em água e pode apresentar reações tóxicas devido as diluições, outro exemplo é o carbonato de bário que acaba sendo insolúvel em água porém possui solubilidade quando em contato com o estômago trazendo então efeitos nocivos ao organismo como hipocalemia, e riscos cardiovasculares (Savazzi, 2008).

A liberação do bário também ocorre através da disposição de resíduos provenientes da produção de fogos de artificio, fabricação de vidro e o uso de defensivos agrícolas. O aumento do bário no ambiente está proporcionalmente ligado as explorações intensas que são feitas nas reservas minerais, e assim ocorre a liberação de resíduos que se encontrarão dispersos ao redor do solo explorado (Savazzi, 2008).

Diferentes metodologias são utilizadas para o tratamento de solos contaminados, como destaque temos a biorremediação que tem como intuito utilizar organismos vivos como micro-organismos e plantas. Na área da biorremediação 
encontra-se a fitorremediação, uma técnica direcionada ao tratamento com o uso plantas, que tem como destaque as suas vantagens por ser eficiente e ter um baixo custo (Pires, Souza, Silva, Procópio\& Ferreira, 2003; Martins, 2009).

Algumas pesquisas científicas vêm mostrando o potencial de diversas espécies de vegetais para o tratamento de áreas contaminadas, com o auxílio da biotecnologia, avanços vêm sendo obtidos para limpeza de áreas poluídas. Os vegetais apresentam inúmeros princípios ativos importantes, com isso diversas pesquisas envolvendo compostos antioxidantes obtidos de extratos vegetais tem sido desenvolvida devido à grande importância na prevenção de desencadeamento das reações oxidativas (Andreo \& Jorge, 2006; Shahidi, Alasalvar \& Pathiranças, 2007; Lamego \& Vidal, 2007; Balunas et al., 2006; Varanda, 2006).

Dentre esses princípios ativos estão os flavonoides, onde se apresentam entre os compostos fenólicos mais abundantes em vegetais, estando concentrados na parte aérea das plantas, como flores e frutos (Perez-Vizcaino \& Fraga, 2018), esses subdividem-se em diferentes classes e as principais são os flavonóis, flavona, flavanonas, antocianinas, flavanas. Os flavonoides são capazes de eliminar radicais livres, inibir enzimas envolvidas na produção de radicais livres e até mesmo regenerar (limitada) as membranas celulares, porém, sua síntese não ocorre em seres humanos (Bobbio\& Bobbio, 2003; Flambó, 2013), além de destacarem-se pelas atividades anti-inflamatória, antiplaquetária, antitrombótica, cardioprotetora, bem como outras funções relacionadas a patologias como a obesidade, diabetes, osteoporose, degeneração hepática e renal, doenças geriátricas, entre outras (Colizzi, 2019; Karak, 2019; Ribeiro et al., 2019).

A quercetina é um dos flavonoides encontrados em maior quantidade na dieta humana e isso acontece por apresentase em abundância nas frutas, chás e verduras, por isso esse tipo de flavonoide chega a ser capaz de desenvolver diversas atividades no organismo humano como: anticarcinogênica, antibacteriana, antifúngica, protetores cardiovasculares, hepáticos e da filtração renal (Simões et al., 2013), além de apresentar-se como uma potencial droga natural no tratamento das dislipidemias (Moura; Araújo; Sousa, 2020).

Devido as diferentes maneiras de liberação e o aumento da exposição do Bário este trabalho foi desenvolvido a fim de avaliar a atividade antibacteriana, antifúngica e citoprotetora da quercetina frente a ação tóxica do cloreto de bário.

\section{Metodologia}

O presente estudo corresponde a uma pesquisa laboratorial aplicada, de natureza experimental e com abordagem quantitativa, mediante coleta de dados numéricos e análise estatística (Pereira, Shitsuka, Parreira \& Shitsuka, 2018).

\subsection{Substâncias e micro-organismos}

O flavonoide Quercetina foi adquirido na Sigma ${ }^{\circledR}$ (St. Louis, USA). O cloreto de bário foi adquirido da Vetec Química Fina LTDA. Os micro-organismos utilizados foram a bactéria Escherichia coli 06 (EC 06) e a Candida albicans 40006 (CA INCQS 40006), fornecidas pelo Laboratório de Micologia da Universidade Federal da Paraíba - UFPB e pela FIOCRUZ por meio do Instituto Nacional de Controle de Qualidade em Saúde (INCQS), e cedidos pelo Laboratório de Microbiologia e Biologia Molecular (LMBM- URCA) e Laboratório de Micologia Aplicada do Cariri (LMAC- URCA), respectivamente.

\subsection{Concentração inibitória mínima (CIM)}

A Concentração Inibitória Mínima (CIM) foi realizada pelo método de microdiluição em caldo. Para a leitura dos ensaios com a bactéria, uma solução de resazurina sódica e água destilada foram adicionadas em cada cavidade $(20 \mu \mathrm{L})$ e as placas foram incubadas por $1 \mathrm{~h}$ em temperatura ambiente. Para revelação da CIM considerou-se como inibição do crescimento os poços que permaneceram com a coloração azul e não inibição os que obtiveram coloração vermelha, quando avaliado para 
bactéria, ou a ausência de turvação no meio, quando avaliado para fungos. A Concentração Inibitória Mínima (CIM) foi definida como a menor concentração capaz de inibir o crescimento bacteriano e fúngico nas cavidades da placa de microdiluição conforme detectado macroscopicamente (Nccls, 2003; Javadpour et al., 1996).

\subsection{Avaliação do potencial citoprotetor em bactéria e fungo contra cloreto de bário}

Para a avaliação do efeito protetor da quercetina ao cloreto de bário, foram preparados eppendorfs contendo concentração sub-inibitória da amostra (128 $\mu \mathrm{g} / \mathrm{mL}$ ) e suspensões de $10^{5} \mathrm{UFC} / \mathrm{ml}$ de Escherichia coli 06 (EC 06) e Candida albicans (CA 40006) em meio TRIS M9. A solução foi distribuída nas cavidades da placa de microdiluição. Logo em seguida foi adicionado $100 \mu \mathrm{L}$ do cloreto de bário na primeira cavidade seguindo com sucessivas microdiluições até a penúltima cavidade. A concentração do metal variou de $10 \mathrm{~mm}$ a $0,00244 \mathrm{~mm}$ as placas de microdiluição foram incubadas por $48 \mathrm{~h}$ a 37 ${ }^{\circ} \mathrm{C}$, em estufa (Sobral- Souza et al., 2018, adaptado).

Em seguida, as concentrações bactericidas mínimas (CBM) e fungicidas mínimas (CFM) foram determinadas como a menor concentração capaz de matar toda a população dos micro-organismos. Foram utilizadas placas de Petri com Agar Heart Infusion (HIA) para transferência das soluções incubadas em placas de microdiluição. Uma alíquota de cada poço da placa de microdiluição foi subcultivada em placas de HIA. Após 24 horas de incubação a $35 \pm 2{ }^{\circ} \mathrm{C}$, foi realizada a leitura, com a finalidade de observação do crescimento das colônias. As leituras da CBM e CFM foram realizadas com base no crescimento dos controles microbianos, sendo considerada CBM e CFM, a menor concentração do extrato e frações que inibiu o crescimento visível do subcultivo (Shadomy, Espinel-Ingroff \& Cartwright, 1985).

\subsection{Ensaio antioxidante dpph}

Foram preparadas soluções com diferentes concentrações de quercetina $(250,125,50,25,10$ e $5 \mu \mathrm{g} / \mathrm{mL})$ em triplicata. Em um tubo foram misturados $100 \mu \mathrm{L}$ da solução da amostra e 3,9 mL de solução de 2,2-difenil-1-picril-hidrazila (DPPH) $0,06 \mathrm{mM}$ e homogenizado com agitador de tubos, procedimento realizado em ambiente escuro. Para o branco, a amostra foi substituída por $100 \mu \mathrm{L}$ de metanol. A curva padrão foi determinada realizando leituras no mesmo comprimento de onda (515 $\mathrm{nm}$ ), porém com soluções de DPPH em diferentes concentrações (10 $\mu \mathrm{M}, 20 \mu \mathrm{M}, 30 \mu \mathrm{M}, 40 \mu \mathrm{M}, 50 \mu \mathrm{M}$ e $60 \mu \mathrm{M})$, sendo o branco determinado por metanol (Sánchez-Moreno, Larrauri \& Saura-Calixto, 2002; Rufino et al., 2007)

Todas as leituras foram realizadas em triplicata, e com a média de dados obtidos, a diferença de absorbância entre as amostras e o controle negativo foi calculado, e o percentual das atividades antioxidantes (AA) obtidos por regressão linear para cada fase, obtendo-se assim a concentração da amostra que promove a redução para metade de $50 \%$ da concentração inicial de DPPH, respectivamente, como definido Concentração Eficaz $\left(\mathrm{CE}_{50}\right)$ (Nascimento et al., 2017).

\subsection{Análises estatísticas}

Todas as determinações foram realizadas em triplicata e os resultados dos ensaios microbiológicos foram analisados através do cálculo das médias geométricas, pela metodologia de Two-way Anova e pos test de Bonferroni, utilizando software Graphpad Prism, versão 7.0 e a análise do teste químico foi realizado através da Anova de One- way.

\section{Resultados e Discussão}

\subsection{Concentração inibitória míniima}

Conforme os resultados expressos na Tabela 1, a quercetina demonstrou resultados análogos tanto para atividade antibacteriana quanto para atividade antifúngica apresentando CIM $\geq 1024 \mu \mathrm{g} / \mathrm{mL}$, mostrando assim que a substância nesse modelo não apresentou atividade bacteriana e fúngica. 
Devido ao aumento da resistência bacteriana à diversas classes de medicamentos, o estudo de plantas com efeito antibacteriano e fungicida vem se destacando por conta dos resultados expressos e pela eficácia do seu potencial de ação (Walker et al., 2009).

Em estudo realizado por Aquino et al. (2015) comprovam que a quercetina apresentou resultados irrelevantes tendo como resultado CIM $\geq 1024 \mu \mathrm{g} / \mathrm{mL}$ a atividade antibacteriana desse estudo foi realizada frente a cepas de Escherichia coli; Staphylococcus aureus e Pseudomonas aeruginosa.

Pesquisas realizadas por Alves et al (2014) mostra que a quercetina não apresentou resultados satisfatórios como antifúngico frente a cepas de Candida, podendo apresentar uma maior resistência contra a Candida tropicalis, apresentando $\mathrm{CIM} \geq 0,625 \mathrm{mg} / \mathrm{mL}$.

Camargo e Raddi, (2008) menciona que a quercetina pode apresentar discrepâncias de resultados em testes de atividade antibacteriana e isso pode acontecer por fatores como a utilização de diferentes tipos de cepas de bactérias e relacionado a metodologia que foi utilizada.

\subsection{Atividade antioxidante pelo método de dpph}

O valor da atividade antixiodante da quercetina realizado pela metodologia de sequestro de radical livre DPPH está representado na Tabela 1, podendo ser observado através da $\mathrm{CE}_{50}$. Quanto mais baixo for o valor da $\mathrm{CE}_{50}$ maior é a potência antioxidante da substância. Observa-se assim que a quercetina apresenta capacidade antioxidante mais efetiva quando comparado ao seu controle, o ácido ascórbico.

A atividade antioxidante de compostos fenóis como a quercetina deriva da sua capacidade de oxido-redução atuando diretamente na absorção e neutralização dos radicais livres que são gerados pelo metabolismo celular ou por fonte exógena, essa proteção antioxidante irá interferir diretamente na formação de lesões e perda da integridade celular (Pereira \& Cardoso, 2012).

Tabela 1 - Atividade Sequestrante calculada em termos de $\left(\mathrm{CE}_{50}\right)$ do composto no teste de DPPH.

\begin{tabular}{|c|c|c|}
\hline Teste & Quercetina & Ácido Ascórbico \\
\hline DPPH & $8,31 \pm 0,09$ & $23,82 \pm 2,10$ \\
\hline
\end{tabular}

Fonte: Dados da pesquisa (2019).

Valores expressos como média \pm erro padrão da média.

Estudos realizados por Alves e Kubota (2013) diz que a atividade antioxidante da quercetina pode acontecer de duas distintas formas sendo elas o sequestro de radicais livres ou através da sua atividade quelante de metal mostrando que quando comparado ao ácido ascórbico seus resultados apresentaram maior eficácia.

Contudo Xu, Hu, Wang e Cui (2019) mostra na sua pesquisa que a quercetina a apresenta uma forte atividade antioxidante, isso acontece devido a sua atividade exercida em vias de transdução de sinal, de espécies reativas de oxigênio, e por exercer efeitos frente a atividades enzimáticas.

\subsection{Concentração bactericida mínima e fungicida mínima (cbm e cfm)}

O Gráfico 1 apresentado abaixo mostra a associação da quercetina ao cloreto de bário a fim de analisar a ação desse composto frente ao teste de CBM e CFM. Os resultados mostram que a quercetina apresentou resultado citoprotetor satisfatório quando associado a bactéria (modulação) contra a toxicidade do cloreto de bário, mostrando assim um efeito 
sinérgico. No entanto, a substância não apresentou efeito citoprotetor significativo quando associado ao cloreto de bário frente a linhagem fúngica.

Gráfico 1: Atividade citoprotetora da quercetina associada ao cloreto de bário contra a ação do cloreto de bário.

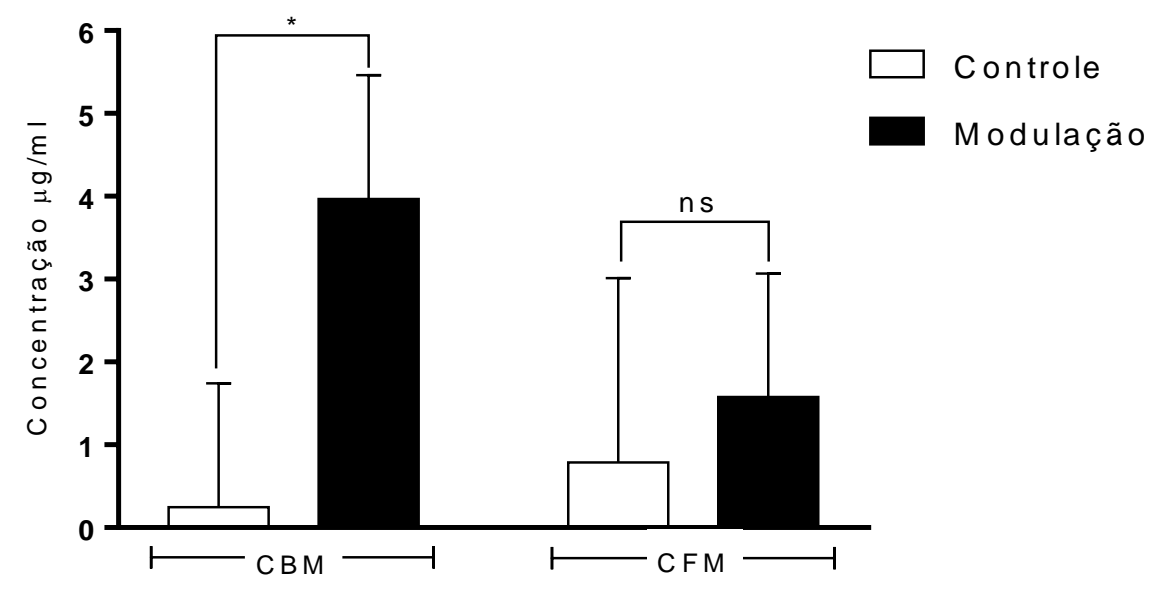

Legenda: Controle: bactéria e fungo+ cloreto de bário; Modulação: quercetina+ cloreto de bário+ bactéria e fungo .Fonte: Dados da pesquisa (2019).

Em estudo realizado por Behling (2004) foi possível observar que a quercetina tem a capacidade de apresentar atividade citoprotetora contra o ataque de radicais livres inibindo o sistema enzimático capaz de produzi-los, a mesma também apresenta a capacidade de quelante e estabilizadora de ferro através da indução de enzimas antioxidantes como a metalotioneína que é uma proteína queladora.

De acordo com Silver e Hobmam (2007), os micro-organismos apresentam resistência à maioria dos metais, ocorrendo normalmente por sistemas de efluxo capazes de remover os metais do citoplasma ou quelação intra ou extracelular do xenobiótico.

Dados da literatura mostraram que a quercetina não demonstrou atividade antifúngica quando testada contra as linhagens padrão de C. albicans ATCC 40227 e C. krusei ATCC 6538 (Veras et al., 2011).

Morais- Braga (2013) relata que os micro-organismos apresentam as suas especificidades genéticas e isso é considerável para explicar a diferença da atividade nos resultados apresentados anteriormente.

\section{Considerações Finais}

Os resultados obtidos neste estudo são pioneiros e o primeiro associando a ação citoprotetora da quercetina contra o cloreto de bário. O estudo indica que o flavonoide quercetina apresenta-se como uma possível alternativa de produto natural com efeitos de proteção celular, provavelmente, devido à atividade antioxidante desta substância. O efeito citoprotetor foi verificado no modelo procariótico de bactéria, quando a quercetina foi associada ao cloreto de bário em baixas concentrações promovendo assim o crescimento da cepa bacteriana. No entanto, não foi capaz de reverter a toxicidade causada pelo cloreto de bário quando utilizada em modelo eucariótico, em associação a cepa fúngica.

Embora o cloreto de bário seja um agente citotóxico em diversas espécies de organismos, percebeu-se que seu complexo com a quercetina pode atenuar estes efeitos. Os testes de toxicidade, utilizando micro-organismos e vegetais, constituem recurso prático de boa sensibilidade na indicação qualitativa da presença de substâncias citoprotetoras que podem ser usadas no combate aos danos ambientais, produzidos pelo acúmulo de metais tóxicos. 
Estes resultados abrem a possibilidade para o desenvolvimento de estudos complementares e a possível aplicação em ambientes contaminados, mais estudos são necessários para observar o efeito de sua aplicação em áreas poluídas, utilizando por exemplo, outros modelos de organismos para avaliar o seu comportamento citoprotetor.

\section{Agradecimentos}

Os autores agradecem ao Centro Universitário Dr. Leão Sampaio (UNILEÃO), ao Laboratório de Microbiologia e Biologia Molecular (LMBM- URCA) e ao Laboratório de Micologia Aplicada do Cariri (LMAC- URCA).

\section{Referências}

Alves, C. T., Ferreira, I., Barros, L., Silva, S., Azenedo, J., \& Henquiques, E. M. (2014). Antifungal activity of phenolic compounds identified in flowers from North Eastern Portugal against Candida species. Future Microbiology, 9(2), 139-146. 10.2217 / fmb.13.147.

Alves, E., \& Kubota, E. H. (2013). Conteúdo de fenólicos, flavonoides totais e atividade antioxidante de amostras de própolis comerciais. Ciências Farmacêuticas Básica e Aplicada, Santa Maria, 1(34), 37-41. https://rcfba.fcfar.unesp.br/index.php/ojs/article/view/233

Andreo, D., \& Jorge, N. (2007). Antioxidantes naturais: Técnicas de extração. Boletim do Centro de Pesquisa de Processamento de Alimentos. $10.5380 /$ cepv24i2.7489.

Aquino, P., Gomes, F. F., Pereira, N., Nascimento, E., Martin, A., Veras, H., Oliveira, C., Ferreira, S., Leandro, L., Silva, M., \& Menezes, I. (2016). Avaliação da atividade anti-inflamatória tópica e antibacteriana do extrato metanólico das folhas de Sideroxylon obtusifolium. Acta Biológica Colombiana, 21(1), 131140. doi:10.15446/abc.v21n1.48170.

Balunas, M. J., Jones, W. P., Chin, Y. W., Mi, Q., Farnsworth, N. R., Soejarto, D. D., Cordell, G. A., Swanson, S. M., Pezzuto, J. M., Chai, H. B., \& Kinghorn, A. D. (2006). Relationships between inhibitory activity against a cancer cell line panel, profiles of plants collected, and compound classes isolated in an anticancer drug discovery project. Chemistry \& biodiversity, 3(8), 897-915. 10.1002/cbdv.200690092.

Behling, E., Sendão, M., Francescato, H., Antunes, L., \& Bianchi, M. L. (2004). Flavonóide quercetina: aspectos gerais e ações biológicas. Alimentos e Nutrição. 15(3), 285- 292. https:// www.researchgate.net/publication/49599688

Bobbio, F. O. \& Bobbio, P. A. (2003). Introdução à Química dos Alimentos. (3a ed.), Varela.

Braga, M. F. B. M., Sales, D. L., Carneiro, J. N. P., Oliveira, O. P., Albuquerque, R. S., Brito, D. I. V., Figueiredo, F. G., Leite, N. F., Tintino, S. R., \& Coutinho, H. D. M. (2013). Efeito antifúngico e atividade moduladora de Lygodium venustum SW. Revista Ouricuri, 3, 146-159. https://revistas.uneb.br/index.php/ouricuri/article/view/6426.

Camargo, R. (2008). Efeito da quercetina sobre o crescimento e atividade hemolítica de Staphylococcus aureus. Araraquara: Revista Eletrônica de Farmácia, 5(3). $0.5216 /$ ref.v5i3.12366

Colizzi, C. (2019). The protective effects of polyphenols on Alzheimer's disease: a systematic review. Translational Research \& Clinical Interventions, 5, 184196. $10.1016 /$ j.trci.2018.09.002.

Lima, E. S. A., Amaral- Sobrinho, N. M. B., Magalhães, M. O. L., Guedes, J. N. \& Zont, E. (2012). Absorção de bário por plantas de arroz (Oryza sativa L.) e mobilidade em solo tratado com baritina sob diferentes condições de potencial redox. Quimica Nova, Seropédica, 35 (9), 1746-1751. 10.1590/S010040422012000900008

Flambó, D. F. A. L. P. (2013). Atividades biológicas dos flavonoides: Atividade antimicrobiana (Dissertação de Mestrado). Universidade Fernando Pessoa, Porto, Portugal.

Javadpour, M. M., Juban, M. M., Lo, W. C., Bishop, S. M., Alberty, J. B., Cowell, S. M., Becker, C. L., \& McLaughlin, M. L. (1996). De novo antimicrobial peptides with low mammalian cell toxicity. Journal of medicinal chemistry, 39(16), 3107-3113. 10.1021/jm9509410.

Karak, P. (2019). Biological activities of flavonoids: an overview. International Journal of Pharmaceutical Sciences and Research, 10(4), 1567-1574. doi: 10.13040 / IJPSR.0975-8232.10 (4) .1567-74

Lamego; V. (2007). Fitorremediação: plantas como agentes de despoluição? Revista de ecotoxicologia e meio ambiente. 17, 9-18. doi:10.5380/pes.v17i0.10662

Lima, A. L. (2012). Amenizantes em solo contaminado com bário. (Tese de Doutorado). Universidade Estadual Paulista "Júlio de Mesquita Filho", Jaboticabal, São Paulo.

Martins, A. L. S. (2009). Remoção de Chumbo e Bário de um efluente aquoso via flotação por ar dissolvido. (Tese de Doutorado). Universidade Federal Rural do Rio de Janeiro Instituto de Tecnologia Programa de Pós-graduação em Engenharia Química, Rio de Janeiro, Rio de Janeiro.

Moura, M. C. L. d., Araújo, V. L. L. d., \& Sousa, J. A. d. (2020). Análise farmacocinética, toxicológica e farmacodinâmica in silico do flavonoide quercetina isolado das sementes de Bixa orellana 1. Research Society and Development, 9,3. 10.33448 / rsd-v9i3.2242 
Nascimento, E., Rodrigues, F., Costa, W. D., Teixeira, R., Boligon, A. A., Sousa, E. O., Rodrigues, F., Coutinho, H., \& da Costa, J. (2018). HPLC and in vitro evaluation of antioxidant properties of fruit from Malpighia glabra (Malpighiaceae) at different stages of maturation. Food and chemical toxicology: an international journal published for the British Industrial Biological Research Association, 119, 457-463. 10.1016/j.fct.2017.11.042.

Nccls. Norma M27-A2. (2003). Método de Referência para Testes de Diluição em Caldo para Determinação da Sensibilidade à Terapia Antifúngica das leveduras; Norma Aprovada - Segunda Edição. Norma M27-A2 do NCCLS (ISBN 1-56238-469-4). NCCLS, 940 West Valley Road, Suite 1400, Wayne, Pennsylvania 19087-1898 Estados Unidos. https://www.anvisa.gov.br/servicosaude/manuais/clsi/clsi_OPAS1M27-A2.

Pereira, A. S., Shitsuka, D. M., Parreira, F. J., \& Shitsuka, R. (2018). Metodologia da pesquisa científica. UFSM. https://repositorio.ufsm.br/bitstream/handle/1/15824/Lic_Computacao_MetodologiaPesquisa-Cientifica.pdf?sequence=1.

Pereira, R.J., \& Cardoso, M. G. (2012). Metabólitos secundários vegetais e benefícios antioxidantes. (Tese de Doutorado), Universidade Federal do Tocantins, Palmas, Tocantins.

Perez-Vizcaino, F., \& Fraga, C. G. (2018). Research trends in flavonoids and health. Archives of biochemistry and biophysics, 646, 107-112. 10.1016 / j.abb.2018.03.022

Pires, F. R., Souza; C. M., Silva, A. A., Procópio, S. O., \& Ferreira, L. R. (2017). Fitorremediação de Solos Contaminados com Herbicidas. Planta Daninha, 21(2), 335-341. 10.1590/S0100-83582003000200020.

Ribeiro, V. R., Fernandes, I. A. A., Mari, I. P., Stafussa, A. P., Rosseto, R., Maciel, G. M., \& Haminiuk, C. W. I (2019). Bringing together Saccharomyces cerevisiae and bioactive compounds from plants: A new function for well-know biosorbent. Journal of Functional Foods, 60(103433),9. 10.1016/j.jff.2019.103433.

Rufino, M. S. M., Alves, R. E., Brito, E. S., Morais, S. M., Sampaio, C. G., Pérez-Jiménez, J., \& Saura-Calixto, F. D. (2007). Metodologia Científica: Determinação da Atividade Antioxidante Total em Frutas pela Captura do Radical Livre DPPH. Embrapa Agroindústria Tropical, Fortaleza. https://www.embrapa.br/en/busca-de-publicacoes/-/publicacao/426953/metodologia-cientifica-determinacao-da-atividade-antioxidante-total-em-frutas-pelacaptura-do-radical-livre- pph.

Sanchez-Moreno, C., Larrauri, J. A., \& Saura-Calixto, F. (1998). A procedure to meausure the antiradical efficiency of polyphenols. Journal of the Science of Food and Agriculture, 76, 270-276. 10.1002 / (SICI) 1097-0010.

Savazzi, E. A. (2008). Determinação da presença de Bário, Chumbo e Crômio em amostras de água subterrânea coletadas no Aqüífero Bauro. (Dissertação de Mestrado). Universidade de São Paulo- Faculdade de Ciências Farmacêuticas de Ribeirão Preto, Ribeirão Preto, São Paulo.

Shadomy, S., Espinel- Ingroff, A., \& Cartwright, R. (1985). Estudos laboratoriais com agentes antifúngicos: teste de sensibilidade e bioensaio. Em Lennette, E. H., Ballows, A., Hausler, W. J., \& Shadomy, H. J. (Eds.), Manual of Clinic Microbiology (4a ed.). American Society of Microbiology.

Silver, S., \& Hobmam, J. L. (2007). Mercury Microbiology: Resistance Systems, Environmental Aspects, Methylation, and Human Health. Microbiology Monographs 6, 357-370. 10.1007/7171_2006_085

Simões, V., Favarin, L., Cabeza, N., Oliveira, T. D., Fiorucci, A. R., Stropa, J., Rodrigues, D., \& Cavalheiro, A. (2013). Síntese, caracterização e estudo das propriedades de um novo complexo mononuclear contendo quercetina e íon Ga (III). Química Nova, 36, 495. 10.1590/S0100-40422013000400002.

Sobral-Souza, C. E., Silva, A. R., Leite, N. F., Rocha, J. E., Sousa, A. K., Costa, J. G., \& Coutinho, H. D. (2019). Psidium guajava bioactive product chemical analysis and heavy metal toxicity reduction. Chemosphere, 216, 785-793. 10.1016/ j. chemosphere.2018.10.174

Varanda, E. A. (2006). Atividade mutagênica de plantas medicinais. Revista de Ciências Farmacêuticas Básica e Aplicada, 27, 1-7. http://repositorio.unesp.br/bitstream/handle/11449/69326/2-s2.0-34047159313.

Veras, H., Santos, I., Santos, A., Fernandes, C., Leite, G., Souza, H... \& Coutinho, H. (2011). Comparative evaluation of antibiotic and antibiotic modifying activity of quercetin and isoquercetin in vitro. Current Topics in Nutraceutical Research, 9, 25-30. https://www.researchgate.net/publication/279545279.

Walker, C. I. B.,Zanotto, C. Z., Ceron, C. S., Pozzatti, P., Alves, S. H., \& Manfron, M. P. (2009). Atividade Farmacológica e Teor de Quercetina de Mirabilis jalapa L. Latin American Journal of Pharmacy, 28(2), 241-246. http://sedici.unlp.edu.ar/handle/10915/7752.

Xu, D., Hu, M. J., Wang, Y. Q., \& Cui, Y. L. (2019). Antioxidant Activities of Quercetin and Its Complexes for Medicinal Application. Molecules, 1123(24), 1-15. 10.3390/moléculas24061123. 